\title{
Porous Flow in Particle-Based Fluid Simulations
}

\author{
Toon Lenaerts* \\ Katholieke Universiteit Leuven
}

\author{
Bart Adams ${ }^{\dagger}$ \\ Stanford University \\ Katholieke Universiteit Leuven
}

\author{
Philip Dutré $\ddagger$ \\ Katholieke Universiteit Leuven
}
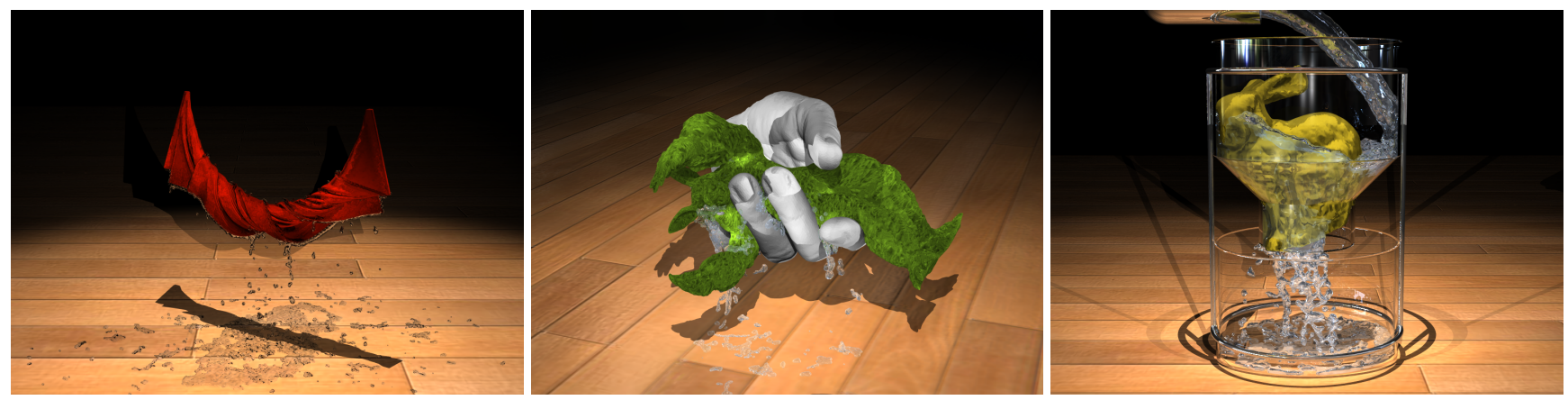

Figure 1: Three examples illustrating porous flow in objects: Wringing fluid out of a towel, squeezing fluid out of a deformable Armadillo, and altering a porous Bunny's material properties by pouring water on it.

\begin{abstract}
This paper presents the simulation of a fluid flowing through a porous deformable material. We introduce the physical principles governing porous flow, expressed by the Law of Darcy, into the Smoothed Particle Hydrodynamics (SPH) framework for simulating fluids and deformable objects. Contrary to previous SPH approaches, we simulate porous flow at a macroscopic scale, making abstraction of individual pores or cavities inside the material. Thus, the number of computational elements is kept low, while at the same time realistic simulations can be achieved. Our algorithm models the changing behavior of the wet material as well as the full two-way coupling between the fluid and the porous material. This enables various new effects, such as the simulation of sponge-like elastic bodies and water-absorbing sticky cloth.
\end{abstract}

CR Categories: I.3.5 [Computer Graphics]: Computational Geometry and Object Modeling_-Physically based modeling

Keywords: porous flow, absorption, smoothed particle hydrodynamics, deformables, particle fluids

\section{Introduction}

Modeling fluid behavior manually quickly becomes infeasible and typically, one resorts to physically-based simulation algorithms to

\footnotetext{
*e-mail: toon.lenaerts@cs.kuleuven.be

†e-mail: barta@stanford.edu

‡e-mail: philip.dutre@cs.kuleuven.be
}

\section{ACM Reference Format}

Lenaerts, T., Adams, B., Dutré, P. 2008. Porous Flow in Particle-Based Fluid Simulations. ACM Trans. Graph. 27, 3, Article 49 (August 2008), 8 pages. DOI = 10.1145/1360612.1360648 http://doi.acm. org/10.1145/1360612.1360648.

\section{Copyright Notice}

Permission to make digital or hard copies of part or all of this work for personal or classroom use is granted without fee provided that copies are not made or distributed for profit or direct commercial advantage and that copies show this notice on the first page or initial screen of a display along with the full citation. Copyrights for components of this work owned by others than ACM must be honored. Abstracting with credit is permitted. To copy otherwise, to republish, to post on servers, to redistribute to lists, or to use any component of this work in other works requires prior specific permission and/or a fee. Permissions may be 2 Penn Plaza, Suite 701, New York, NY 10121-0701, fax + (212) 869-0481, or permissions@acm.org.

(1)

http://doi.acm.org/10.1145/1360612.1360648 enable fluid effects in computer graphics applications. Although great progress has been made in fluid-rigid [Carlson et al. 2004] and fluid-deformable [Guendelman et al. 2005] animations, the focus has been mostly on the coupling between fluids and impenetrable solid objects or shells. Yet not all objects are made out of impenetrable solid material; most materials are porous when viewed at the appropriate scale. These materials absorb and diffuse fluid through their body upon interaction, which affects the physical properties of the deformable body. E.g., a soaked spongy ball thrown at a wall reacts differently as opposed to a dry one, or wet cloths stick to surfaces due to surface tension forces of the present fluid. To include these effects in computer graphics, not only the absorption and emission processes need to be modeled, but also the fluid diffusion within the porous material, as well as the changing material properties of the deformable objects.

In this work we present a novel particle method based on Smoothed Particle Hydrodynamics (SPH) to simulate fluid-absorbent deformable objects and their changing behavior. Our framework for fluid and deformable solid and shell simulations unifies the prior work of [Müller et al. 2003; Müller et al. 2005; Solenthaler et al. 2007; Becker and Teschner 2007]. We extend it to simulate the wetting of objects; not only at the surface, but also throughout the volume of the object. Secondary effects such as the weakening and sinking of fluid absorbent objects follow immediately from the changing physical particle properties.

The main contribution of our method is the treatment of the pores at a macroscopic scale. Previous SPH methods modeling the porous body at the pore scale [Sawley et al. 1999; Morris et al. 1997; Zhu et al. 1999] suffer from long simulation times and memory usage, making them not very practical and too detailed for computer graphics animations. In contrast, we reuse the particle representation of the deformable objects and add additional properties to model the diffusion process within porous materials. Collocating the elastic and porous material properties facilitates modeling of changing material behavior and results in a lightweight particle representation that can easily be incorporated in existing animation algorithms.

Previous Work Fluid animations have received considerable attention in recent years. As opposed to Eulerian models [Foster and Metaxas 1996; Foster and Metaxas 1997; Stam 1999; Foster and 
Fedkiw 2001], particle-based models are a popular Lagrangian way of solving fluid flow. These are typically based on the Smoothed Particle Hydrodynamics (SPH) method (see [Monaghan 2005] for a good overview). SPH was introduced in the graphics community by [Desbrun and Gascuel 1996] to simulate highly deformable objects and popularized by [Müller et al. 2003; Müller et al. 2005] for simulating multiple fluids at interactive rates. Premože et al. [2003] concurrently introduced the Moving Particle Semi-Implicit (MPS) method in computer graphics as an alternative for solving particle-based fluids. Clavet et al. [2005] simulated viscoelastic fluids and their interaction with rigids. Interactive simulations and visualizations of rivers were made possible by [Kipfer and Westermann 2006]. Performance was increased by adaptively sampling the fluid volume [Desbrun and Cani 1999; Adams et al. 2007], while [Kolb and Cuntz 2005; Hegeman et al. 2006; Harada et al. 2007] proposed SPH implementations that exploit the capabilities of current graphics hardware. The simulation of fluids and solid objects was first unified by [Keiser et al. 2005] and later extended by [Solenthaler et al. 2007]. None of these methods are able to simulate the absorption process or the porous flow through a body.

The study of fluid flow through porous media is scattered throughout many fields of science. A good overview of the physics of flow through porous media is given by [Scheidegger 1957]. Bear [1972] was the first to present and use the continuum approach in modeling flow and transport phenomena. A more recent presentation on the subject was published by [Hilfer 1996]. The use of SPH for modeling flow in porous media at a pore scale was concurrently investigated for predictive simulations by different authors. Sawley [1999] presented an SPH framework in which porous media are modeled by fixed particles in the fluid domain. In a series of papers Zhu, Fox and Morris extended SPH to allow incompressible porous flow [Morris et al. 1997; Zhu et al. 1999] and describe diffusion in spatially periodic porous media [Zhu and Fox 2001]. A Riemann-SPH method was introduced by [Berry et al. 2004] to simulate contaminant transport and deposition in porous media. While these approaches result in accurate solutions, they are limited by the computational costs for practical problems as Zhu et al. [1999] concluded. The problem was alleviated by Morris et al. [1999] as they showed that SPH can be parallelized to simulate flow through porous media. However, the porous flow simulations are performed on the pore-scale and are, as such, still prohibitively expensive for use in a computer graphics context. However, [Chu and Tai 2005] showed how ink can be dispersed in absorbent paper using a lattice Boltzmann approach on graphics hardware.

Overview Section 2 summarizes our particle framework for the simulation of fluids, solid objects and shells. In Section 3 we describe how porous materials such as sponges and cloth can be modeled and how fluid diffusion in deformable objects is simulated, resulting in changing material properties that affect the simulation. Section 4 describes the coupling between our exterior particle fluid simulation and the fluid diffusion process within porous materials. We propose fluid absorption and emission strategies that adequately couple fluids and porous objects. Section 5 discusses the animation and visualization of the (wet) boundary surface associated with the deformable particle volumes. Finally, a discussion of our results is given in Sections 6 and 7 and we conclude and provide avenues for future extensions in Section 8.

\section{Particle Simulation Framework}

We simulate our fluids and deformable objects using SPH methods. The fluid and deformables are sampled and represented as discrete particles $p_{i}$, centered at the position $\mathbf{x}_{i}$, with mass $m_{i}$, volume $V_{i}$ and material density $\rho_{i}=m_{i} / V_{i}$. Continuous properties (such as interaction forces) are derived from the discrete particles using the classical SPH equation ([Monaghan 1992; Monaghan 2005]):

$$
A(\mathbf{x})=\sum_{j} V_{j} A_{j} W\left(\mathbf{x}_{j}-\mathbf{x}, h_{j}\right),
$$

where $W\left(\mathbf{x}_{j}-\mathbf{x}, h_{j}\right)$ is the radially symmetric kernel function and $h_{j}$ is the interaction kernel size (or smoothing length) associated with particle $p_{j}$. We will use the SPH equation extensively in the following to approximate continuous properties from the discrete particle representation.

Our simulation framework unifies prior work on fluid, (deformable) solid and shell simulations. For sake of completeness, we briefly summarize these simulation methods.

Fluid Simulation Our SPH fluid framework is based on the work of [Müller et al. 2003; Müller et al. 2005; Becker and Teschner 2007]. Fluid behavior is modeled by the Navier-Stokes momentum equation $\left(\rho \frac{D \mathbf{v}}{D t}=-\nabla P+\mu \nabla^{2} \mathbf{v}+\rho \mathbf{g}\right)$, where $\mathbf{v}$ is velocity, $P$ is pressure, $\mu$ is viscosity and $\mathbf{g}$ is the gravity. Using SPH, one easily obtains the particle forces [Müller et al. 2003]. Because the absorption and emission of fluid will introduce differently sized particles, we have varying per-particle smoothing lengths $h_{i}$, which are chosen as a multiple (typically 2 to 3 ) of the particle radius. The particle radius $r_{i}$ is obtained from the particle mass $m_{i}$ and the water density $\rho_{i}$ by solving $4 / 3 \pi r_{i}^{3}=m_{i} / \rho_{i}$. We use the shooting-gathering approach of [Desbrun and Cani 1999] to guarantee symmetric forces.

Solid and Shell Simulation Rigid bodies are simulated as described in [Keiser et al. 2006]. The rigid volume is sampled with particles and forces acting on these particles are accumulated to a total force and torque on the body to enforce rigid body motion. Deformable bodies are simulated using SPH as in [Solenthaler et al. 2007]. Elastic forces are computed for each particle using a locally undeformed object condition. Each particle stores distance vectors to its neighboring particles in rest. Displacements from this reference neighborhood translate into a strain $\epsilon$ from which the stress $\sigma$ can be computed using Hooke's law, $\sigma=\mathbf{C} \epsilon$. We extend the framework of [Solenthaler et al. 2007] to allow the simulation of cloth. This is achieved by discarding the orientation of particles in the reference neighborhood and using only the distance between neighboring particles. Doing so appropriately allows for bending, but prohibits material stretching.

Explicit collision handling is added based on the Molecular Dynamics approach of [Bell et al. 2005]. We adopt the method from [Clavet et al. 2005] to describe adhesion forces between fluids and rigid or deformable bodies. Particles are integrated in time using the leap-frog integration scheme. When using variable time steps we revert to a first order forward Euler method.

\section{Simulating Flow in Porous Materials}

\subsection{Modeling Porous Materials}

A porous medium can be modeled at different scales. On the porescale, each of the individual pores or cavities could be carved out of the solid phase. In this paper, we opt for an efficient representation at a macroscopic scale. We remodel rigid or elastic particles as porous particles and let them represent a small porous volume, capable of holding an amount of fluid (Figure 2). This unified representation allows us to use the same particle resolution as for regular solid-fluid simulations. Moreover, it allows modeling changing material behavior when fluid is absorbed by a particle.

A porous particle is completely defined by its porosity and permeability. The porosity $\phi_{i}$ denotes the volume fraction of the interconnected void space at the particle, i.e., $\phi_{i} V_{i}$ denotes the void particle 


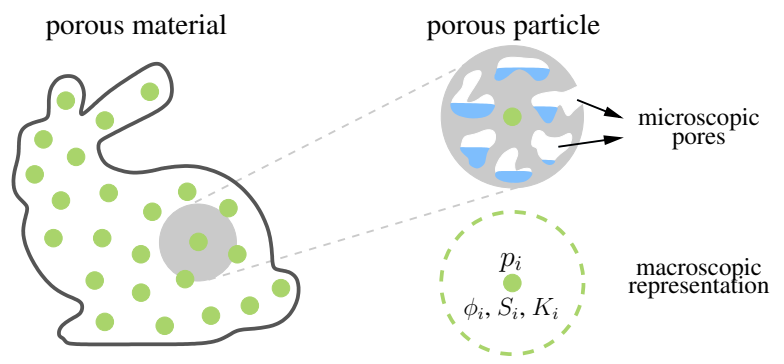

Figure 2: Porous materials are represented at a macroscopic scale. Pores or cavities of a certain region in the volume are not modeled explicitly. Instead, the region is represented at a coarser level by the use of porous particles (right), which have porosity and permeability parameters that characterize the surrounding material.

volume. Hence, a particle $p_{i}$ with porosity $\phi_{i}$ can hold an absorbed fluid mass $m_{p i} \leq \rho^{\text {fluid }} \phi_{i} V_{i}$ which defines the particle's saturation $S_{i}$ :

$$
S_{i}=\frac{m_{p i}}{\rho^{\text {fluid }} \phi_{i} V_{i}} .
$$

Note that $m_{p i}$ denotes the fluid mass in the porous particle, while $m_{i}$ denotes the particle's own unsaturated mass.

The permeability $\mathbf{K}$ of a porous medium characterizes its ability to transmit a certain fluid. $\mathbf{K}$ is a second-order symmetric tensor, which may have non-zero off-diagonal elements if the porous medium is anisotropic. It reflects the actual internal distribution of pores for a certain region in the material. In our work, we focus on isotropic porous materials, hereby reducing the permeability tensor $\mathbf{K}=K \mathbf{I}$ to a scalar. We assign a permeability $K_{i}$ to each porous particle $p_{i}$ and are therefore able to model both homogeneous and heterogeneous porous media.

\subsection{Simulating Porous Flow}

For small-scale fluid dynamics, surface tension (or interfacial tension) is of great importance. At the pore-scale, the entrance of fluid into a pore is facilitated by the surface tension because of a pressure differential between the entering fluid phase and the displaced phase inside the porous medium, which can be air or another fluid. This pressure, called the capillary pressure, makes fluid diffuse to neighboring, less saturated pores. These forces also keep the fluid trapped inside the medium. Since we have no microstructure of the pores, we model the capillary forces acting on the fluid represented in a porous particle as gradients of capillary potentials [Nitao and Bear 1996; Hilfer 2006], which we compute using SPH:

$$
\nabla P_{i}^{c}=\sum_{j} V_{j} P_{j}^{c} \nabla W\left(\mathbf{x}_{j}-\mathbf{x}_{i}, h_{j}\right)
$$

where the capillary potential $P^{c}$ is defined as a pressure function of the saturation $S$ :

$$
P_{i}^{c}=k^{c}\left(1-S_{i}\right)^{\alpha},
$$

with constants $k^{c}$ and $0<\alpha<1$ controlling the strength of the potential. The capillary force of the pore space of a porous particle drops as its saturation increases.

Another important pressure difference can arise when deformations of a porous elastic body change the volume of the pore space. These porosity changes may exert a pressure on the present fluid, causing it to flow out of the squeezed pores. This leads to a variable porosity $\phi_{i}$ depending on the local density of the medium:

$$
\phi_{i}=\phi_{0} \frac{\rho_{0}^{s}}{\rho_{i}^{s}}
$$

where $\phi_{0}$ is the porosity in rest. We compute the pore pressure $P_{i}^{p}$ similar to the equation of state as in [Becker and Teschner 2007]:

$$
P_{i}^{p}=k^{p} S_{i}\left(\left(\frac{\rho_{i}^{s}}{\rho_{0}^{s}}\right)^{\gamma}-1\right) \text {. }
$$

The saturation level $S_{i}$ is added as a factor, motivated by our macroscopic approach: Fluid may flow inside the volume of one porous particle as long as it is not fully saturated.

These pressure differences give rise to the Darcy flux [Darcy 1856], which governs the flow of an incompressible, single-phase fluid through a porous medium. It can be derived from the more general Navier-Stokes equations and relates a pressure gradient $\nabla P$ on a fluid with viscosity $\mu$ and completely filling a porous medium with permeability $K$ to a flux. This Darcy flux is related to the pore velocity $\mathbf{v}_{\mathbf{p}}$ by dividing by the porosity $\phi$ :

$$
\mathbf{v}_{\mathbf{p} i}=-\frac{K_{i}}{\phi_{i} \mu}\left(\nabla P_{i}^{p}-\nabla P_{i}^{c}-\rho \mathbf{g}\right)
$$

This pore velocity field defines an anisotropic diffusion inside the medium. In contrast to the fluid particles outside of the porous medium, we model the fluid flow inside the medium in a Eulerian way using a diffusion process. Fluid mass is diffusing from one porous particle to the next, rather than tracing fluid particles through the medium. The SPH approximation to the diffusion equation [Müller et al. 2005] for the evolution of the absorbed fluid mass $m_{p}$ is:

$$
\frac{\partial m_{p i}}{\partial t}=\sum_{j} d_{i j} V_{j} m_{p j} \nabla^{2} W\left(\mathbf{x}_{j}-\mathbf{x}_{i}, h_{j}\right),
$$

in which we define the diffusion coefficients $d_{i j}$ between two particles proportional to the direction and length of the pore velocity:

$$
d_{i j}=\mathbf{v}_{\mathbf{p} j} \cdot \frac{\mathbf{x}_{j}-\mathbf{x}_{i}}{\left\|\mathbf{x}_{j}-\mathbf{x}_{i}\right\|} S_{j}^{\beta} .
$$

Similar to [Bear 1972; Cuesta et al. 1999; Hilfer 2006], we make the diffusion dependent on the saturation $S_{i}$ of a porous particle. The user defined $\beta>0$ is a parameter to control this flow. It can be interpreted as internal flow, where pores inside the particle volume are filled before flowing to neighboring particles

Finally, the fluid mass is integrated in time using an explicit Euler integration step:

$$
m_{p i} \leftarrow m_{p i}+\Delta t \frac{\partial m_{p i}}{\partial t}
$$

Due to the Eulerian nature of the diffusion process, mass conservation is not automatically guaranteed and the total diffused fluid mass must be explicitly controlled. We do this by explicitly checking the diffusing fluid mass against the free and occupied volumes, $\left(1-S_{i}\right) \phi_{i} V_{i}$ and $S_{i} \phi_{i} V_{i}$ respectively, inside the porous particles.

\subsection{Modeling Changing Material Properties}

Fluid absorbed by a porous object affects its material parameters and hence its behavior. Particles gain weight, implying the solid mass $m_{i}$ and absorbed fluid mass $m_{p i}$ of a porous particle should be used in all computations. The density $\rho_{i}$ of a porous particle is called the bulk density. The bulk rest density becomes:

$$
\rho_{0 i}=\rho_{0}+S_{i} \phi_{i} \rho_{0}^{\text {fluid }},
$$

to account for the saturated pores in the equation of state. The net result for a soaked body is a higher mass and density and thus changed physical behavior as compared to the dry material. 

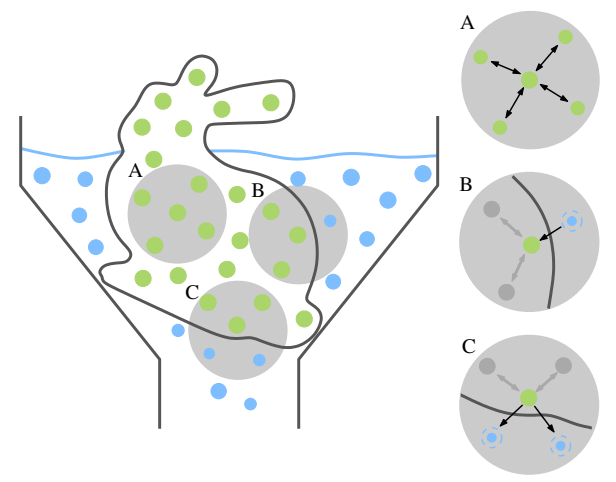

Fluid diffusion within the porous material.

Figure 3: Three different cases for fluid transportation in the presence of porous materials. A: Within the porous object fluid mass is diffused at a macroscale between the porous particles. B: Fluid particles near the porous material are treated as porous particles and mass is taken away from them if fluid is absorbed by the porous material. C: When the porous material emits fluid, mass is added to neighboring fluid particles, which are again treated as porous particles. If, after the diffusion process, more fluid remains to be emitted, new fluid particles will be created in the neighborhood of the porous particle.

When simulating elastic bodies, the fluid pressure also reduces the stress $\sigma_{i}$ in the body:

$$
\sigma_{i}^{e f f}=\sigma_{i}-\eta P_{i}^{p *} \mathbf{I}
$$

Equation 12 is called the effective stress and determines material weakening and volume gain. The $P_{i}^{p *}=k^{p} S_{i}$ represents the pore pressure of the present fluid. Resulting from our experience we added a scaling factor $\eta$ to the equation to control the balance between porous flow (Equation 6) and the influence of the pore pressure on the elastic body.

\section{Porous Medium-Fluid Coupling}

At the contact surface between a porous material and the surrounding fluid, porous particles interact with fluid particles. The surrounding fluid is absorbed using particle deletion and absorbed fluid can be emitted back into the surrounding fluid by dynamic particle creation. We now discuss these absorption and emission processes.

\subsection{Absorption}

Fluid particles near the surface of a porous body may be absorbed due to capillary forces. We use the same diffusion process as described in the previous section by treating the fluid particles at the surface as saturated porous particles with a porosity $\phi_{i}=100 \%$ and saturation $S_{i}=1$ in Equations 3 to 10. These fluid particles are included in the diffusion process and are deleted as soon as all their mass is diffused in the absorbing material (Figure $3 \mathrm{~B}$ ).

The gradual absorption of the fluid particle mass avoids discontinuities that would arise if we would absorb the fluid particles at once and decouples the resolution of the fluid from the resolution of the porous body. However, it introduces variable sized fluid particles in the fluid simulation framework. As discussed above, we employ the shooting-gathering approach of [Desbrun and Cani 1999] to obtain symmetric forces between particles with different smoothing lengths. A variable timestep based on the Courant condition for the smallest $h_{i}$ stabilizes the simulation. A threshold for the particle volume, typically down to $1 \%$ of the original size, is enforced to guarantee a minimum timestep.

\subsection{Emission}

When the pore velocity field $\mathbf{v}_{\mathbf{p}}$ pushes the fluid outside the medium, fluid mass has to be transferred from the porous material to the surrounding fluid (Figure $3 \mathrm{C}$ ). Similarly to the absorption mechanism, we treat fluid particles at the interface as unsaturated porous particles $\left(S_{i}=0\right)$ and let absorbed fluid mass diffuse to the fluid particles as described in Section 3.2. These fluid particles may grow in volume until they reach the normal size of a fluid particle.

If no fluid particles surround the porous material or if additional fluid mass remains to be emitted, new fluid particles are created as follows: The pore velocity field $\mathbf{v}_{\mathbf{p}}$ defines the positions for these new fluid particles for the current timestep. To avoid large pressure forces between neighboring fluid particles, we follow the approach of [Adams et al. 2007] to maximize the distance to existing particles. If there is a particle too close (within a threshold distance), we look for a new position by random sampling around this target position within a sphere with half the particle radius. Ensuring this minimal particle distance results in a more stable particle creation and emission process. Moreover, new fluid particles are usually relatively small at creation and will push neighboring particles aside as they expand due to diffusion in the next timesteps.

\section{Surface Animation \& Visualization}

The surface of the rigid and deformable objects in our simulation framework is defined by a triangle mesh that is animated along with the simulation particles. Neighboring particles are computed for each vertex in the object's undeformed rest shape. The deformation of each vertex can then be efficiently computed from the neighboring particles' deformations using the embedded deformation approach as presented in [Müller et al. 2004a]. The surfaces of the fluid particle volumes are extracted as in [Müller et al. 2003] using Marching Cubes [Lorensen and Cline 1987] and are rendered in POV-Ray.

The appearance of objects changes when water or another fluid is absorbed. In general, wet materials look darker compared to dry materials due to increased light absorption [Twomey et al. 1986]. Although complex models for the rendering of wet surfaces with full subsurface scattering are available [Jensen et al. 1999], we use a simple and intuitive model for visualizing our results. We model the absorption using a darkening factor $\delta(0<\delta<1)$ for wet regions. If $f_{r}^{d r y}$ is the Bidirectional Reflectance Distribution Function (BRDF) of the completely dry material, the BRDF of the wet material, $f_{r}^{\text {wet }}$, is approximated at particle $p_{i}$ by

$$
f_{r i}^{w e t}=S_{i} \phi_{i}\left(\delta f_{r}^{d r y}\right)+\left(1-S_{i} \phi_{i}\right) f_{r}^{d r y},
$$

where $\delta f_{r}^{d r y}$ is the completely wet material and $S_{i} \phi_{i}$ is a measure for the wetness. A continuous BRDF evaluation at the vertex positions $\mathbf{x}$ is obtained using Equation 1:

$$
f_{r}^{w e t}(\mathbf{x})=\sum_{j} V_{j} f_{r j}^{w e t}\left(\mathbf{x}_{j}\right) W\left(\mathbf{x}_{j}-\mathbf{x}, h_{j}\right) .
$$

\section{Results}

We implemented the proposed simulation framework in $\mathrm{C}++$. All simulations are executed on a single processor computer with a $2.93 \mathrm{GHz}$ CPU and $4 \mathrm{~GB}$ of memory.

In all animations we set the capillary strength $k_{c}$ to $15,000 P a$ and $\alpha$ and $\beta$ respectively to 0.1 and 7 . We use a value of $0.01 P a \cdot s$ for the artificial viscosity of water [Monaghan 2005; Becker and Teschner 2007]. Full animations can be seen in the accompanying video. 

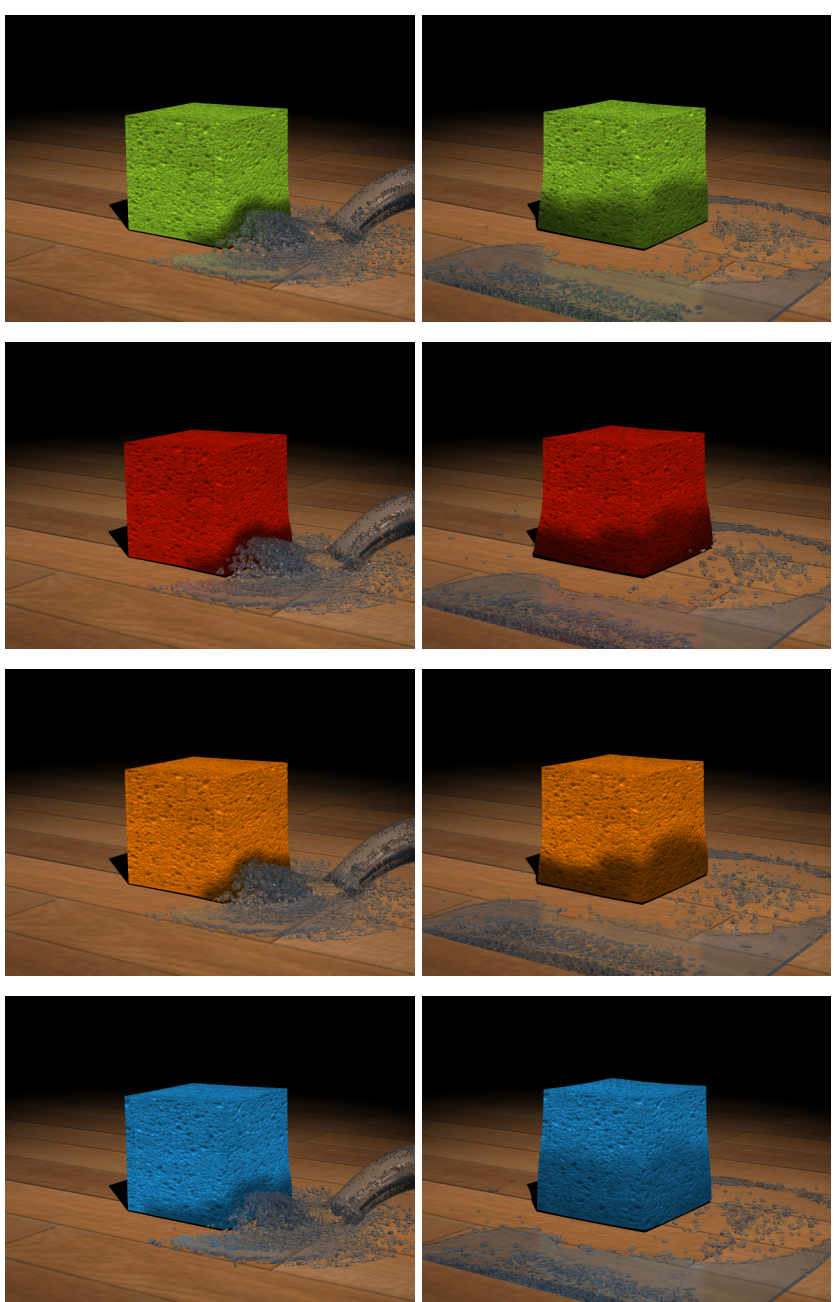

Figure 4: Animations showing the effects of the porous flow parameters by pouring water against a dry cubic sponge. Higher pore pressures affect the volume gain (red sponge), a lower permeability slows the flow down (orange sponge) and a higher capillary pressure pulls the water higher up the sponge (blue sponge).

Figure 4 shows a comparison of the porous flow parameters by pouring water against a dry sponge, measuring $5 \mathrm{~cm} \times 5 \mathrm{~cm} \times$ $5 \mathrm{~cm}$. The green sponge has a $70 \%$ porosity and a permeability of $1 e^{-12} m^{2}$. Notice how the percolated fluid affects the volume and stiffness of the sponge. The pore pressure constant $k^{p}$ is set to $5 e^{4} \mathrm{~Pa}$ for the green sponge, whereas the red sponge has a $k^{p}$ value of $7.5 e^{4} \mathrm{~Pa}$ and thus has a larger volume gain. The orange sponge's permeability is 10 times lower than the one of the green sponge causing the fluid to flow at a slower pace. The twice as high capillary pressure of the blue sponge causes the fluid to rise up higher in the sponge volume. The sponges are simulated using 5000 porous particles, the water is simulated using 25,000 particles.

Figure 5 shows a tank filled with a heterogeneous porous material with varying permeabilities. The porous medium's dimensions measure $10 \mathrm{~cm} \times 10 \mathrm{~cm} \times 10 \mathrm{~cm}$. An S-shaped region in the porous medium has a permeability of $1^{-12} \mathrm{~m}^{2}$, whereas the other regions have a permeability of only $2 e^{-15} \mathrm{~m}^{2}$. The porosity does not vary and amounts to $50 \%$. As expected, the regions of higher permeability saturate first as the fluid flows down the tank. Capillary forces pull the fluid to the right in the lower part of the S-shape. 32,000 particles are used to simulate the porous medium and the fluid on
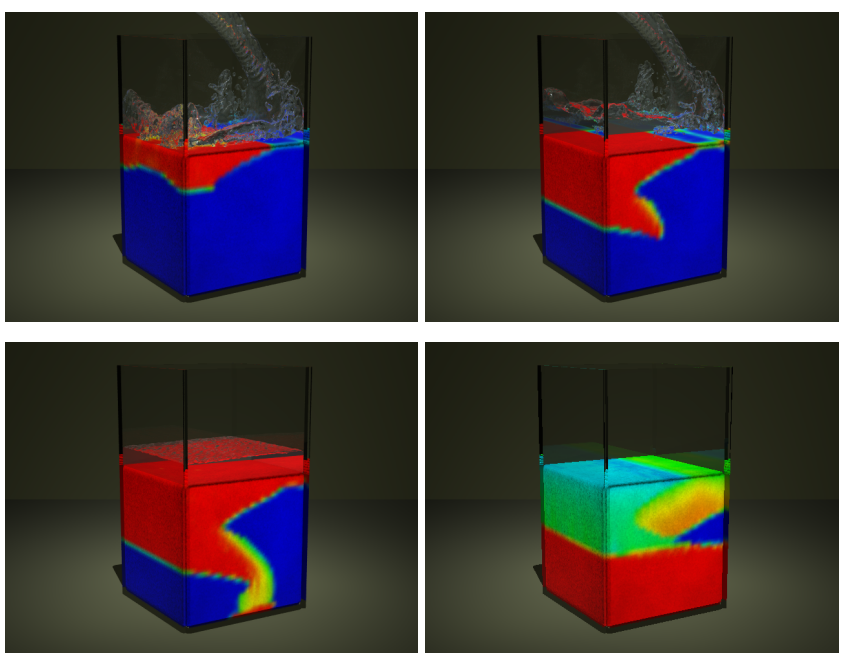

Figure 5: Water flowing through a heterogeneous porous material. An S-shaped region has a 500 times higher permeability, allowing the fluid to reach the bottom more easily. The saturation level is false-color coded ranging from dry (blue) to completely wet (red).

top is simulated by maximum 32,000 particles. The average computation time per frame is 2 minutes.

Figure 6 shows an animation of a wet cloth's tendency to stick to surfaces due to high surface tension forces caused by a small layer of fluid between the surface and the cloth. We simulate this behavior by increasing the adhesion forces between cloth particles and surfaces and make the friction proportional to the saturation level of the porous particle. The result can be seen in the top row of Figure 6 in which a $30 \mathrm{~cm} \times 40 \mathrm{~cm}$ wet velvet cloth is draped around a sphere and starts dripping. The cloth is sampled by a single layer of 20,000 particles that have a uniform porosity of $70 \%$, a permeability of $1 e^{-12} \mathrm{~m}^{2}$ and are $80 \%$ saturated in the beginning of the animation. Notice how the cloth folds and sticks around the sphere due to the present fluid, in contrast to a dry cloth which can be seen in the accompanying video. The bottom row of Figure 6 shows frames from an animation where the same cloth is twisted to wring the fluid out of the cloth. As the cloth folds and tightens, the porosity is reduced and the water naturally flows out of the cloth. Approximately $80 \%$ of the fluid in the porous particles is emitted at the cloth surface, hereby creating 25,000 fluid particles. The computation time per frame was on average 1.5 minutes for both animations.

Figure 7 shows an animation in which water inside a porous Armadillo model is squeezed out. The Armadillo is sampled with 30,000 porous particles of $70 \%$ porosity and saturated for $95 \%$. Capillary forces hold the fluid mass in the porous volume unless the porous particles are deformed (e.g., around the fingers). As the hand closes the volume of the Armadillo is reduced by $15 \%$ on average and the resulting pore pressure pushes the fluid out. Notice how emitted fluid can be absorbed again at a different point. While the hand squeezes the number of particles increases to 20,000 and drops again to 10,000 when the hand stops moving. The computation time was approximately 20 minutes per frame due to a small simulation time step that was required to ensure adequate squeezing behavior avoiding hand-Armadillo penetrations.

As presented in Section 3.3 the fluid pressure in the pores absorbs some of the stress of the pore-elastic body. The resulting behavior of the effective stress is illustrated in Figure 8. A dry Stanford Bunny consisting of 24,000 porous particles is dropped into a funnel. The material is strong enough to support and hold the Bunny above the funnel. As we pour water in the funnel (60,000 particles), 

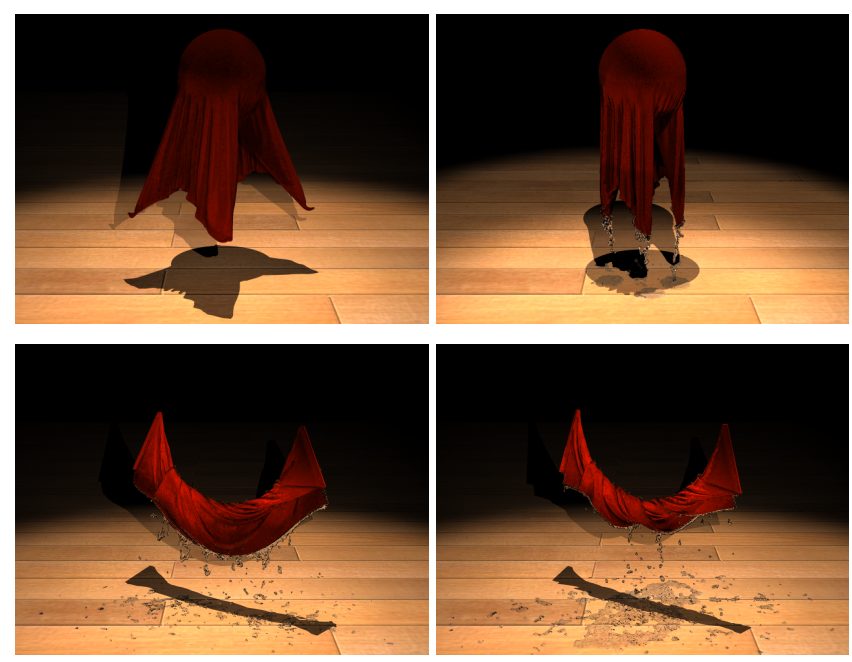

Figure 6: Wet cloth simulations. Top: A wet cloth is draped over a sphere. Water flows down the cloth and forms jets at the tips. Bottom: Twisting a wet cloth causes the fluid to flow out of the reduced pore space.

the Bunny absorbs the water and becomes weaker. Eventually the material is no longer capable of supporting the increased weight causing the Bunny to sag through the funnel into the reservoir below.

\section{Discussion}

Our porous flow model is based on a macroscopic approach in which porous particles represent a volume of solid mass and empty space. The most important advantage of using SPH to model the diffusion process is that we can easily handle absorption and emission at the boundary between the fluid and the deformable body. Porous particles and water particles are treated in a uniform manner which greatly simplifies the algorithm. This advantage will be lost when resorting to a mesh-based method for the diffusion process. Modeling the internal diffusion process using mesh-based approaches should be easy, however, handling the absorption and emission at the interfaces seems to be a non-trivial problem.

In this particle representation fluid can flow from one particle to the next, but flow inside one particle is lost in the representation. We address this by using the saturation level as a scaling factor in Equation 9. This effectively stalls flow in the porous particle volume and hereby achieves the desired wet flow front instead of a very gradual diffusion. Using the equation of state for pore pressure calculations holds the same compressibility issues as for SPH fluid simulations. Therefore we can not guarantee volume preservation of the fluid in a deformable porous body, causing porous flow to start too late when squeezing a porous body using a low pore pressure constant.

Our SPH framework for solving elastic bodies and cloth can be completely decoupled from the porous flow framework. We have chosen for a uniform SPH model, but other techniques for simulating elasticity such as those based on Moving Least Squares shape function approximations (e.g., [Müller et al. 2004a]) might be better suited for certain animations. Next to the effective stress one could also adjust the elastic parameters to model the behavioral changes in the wet material. While Young's modulus generally seems to decrease with increasing water saturation, the behavior of Poisson's ratio seems less clear. Since these adjustments are highly material dependent, we propose to use only the effective stress, which should suffice in most computer graphics applications.
The inclusion of porous flow through bodies requires an extra iteration step over the particles and their neighbors to diffuse the absorbed fluid mass. The capillary and pore pressure and their gradients can be computed in the same steps as the density and density force field respectively [Müller et al. 2003]. However, to compute the pore-velocity field the pressure gradients must be known. This extra iteration over the particles only marginally increases the simulation time. Figure 6 took about $9 \%$ longer in simulation time for the porous cloth compared to the solid cloth (not accounting for the created fluid particles).

In our experience, the shrinking fluid particles can be the main cause of a slow down of the simulation time. Their smoothing length determines the time step and hence the simulation time per frame. In a single simulation time step, most of the computation time is spent on neighborhood queries (as also noted in [Keiser et al. 2006]). We currently use a grid-based acceleration structure similar to [Müller et al. 2003] that works reasonably well. Even though our algorithm introduces differently sized particles, most particles have a uniform size and only a small fraction of particles (typically near the boundary of the porous material) have smaller radii. Using a uniform grid, with grid size based on the largest, most common, support radius therefore only incurs little overhead for the smaller particles. However, we plan to investigate whether a $k$ - $d$ tree would further decrease the time spent on range queries.

For porous objects that only deform a little, the precomputed reference neighborhood can be used to speed up internal diffusion. However, when simulating cloth as in the wringing cloth demo (Figure 6), this would not yield the correct results, as pieces of cloth overlap and touch and particle neighborhoods change significantly during simulation. Here, dynamic neighborhood computation results in the correct diffusion behavior.

\section{Conclusion}

In this paper we have presented an SPH method for simulating fluid flowing through a porous material. Rigid and deformable objects are sampled by particles which represent local porosity and permeability distributions at a macroscopic scale. Our diffusion method for porous flow can easily be incorporated into existing particlebased simulations. Various animations demonstrate new effects due to absorption, such as material weakening, which are not possible with current fluid animation systems.

In future work we would like to extend the porous flow framework to handle multi-phase flow through a porous material. This would allow for simulations with e.g. air-filled pores, which can form bubbles under water.

\section{Acknowledgments}

Toon Lenaerts is funded by a Ph.D. grant of the Institute for the Promotion of Innovation through Science and Technology in Flanders (IWT-Vlaanderen). Bart Adams is funded as a post-doctoral researcher by the Fund for Scientific Research, Flanders, Belgium (F.W.O.-Vlaanderen). We would like to thank the reviewers for their contributing remarks. We also thank Stanford University for making available the Armadillo and Bunny models.

\section{References}

Adams, B., Pauly, M., Keiser, R., And Guibas, L. J. 2007. Adaptively sampled particle fluids. In SIGGRAPH '07: ACM SIGGRAPH 2007 papers, ACM, New York, NY, USA, 48.

Bear, J. 1972. Dynamics of Fluids in Porous Media. Dover Publications. 

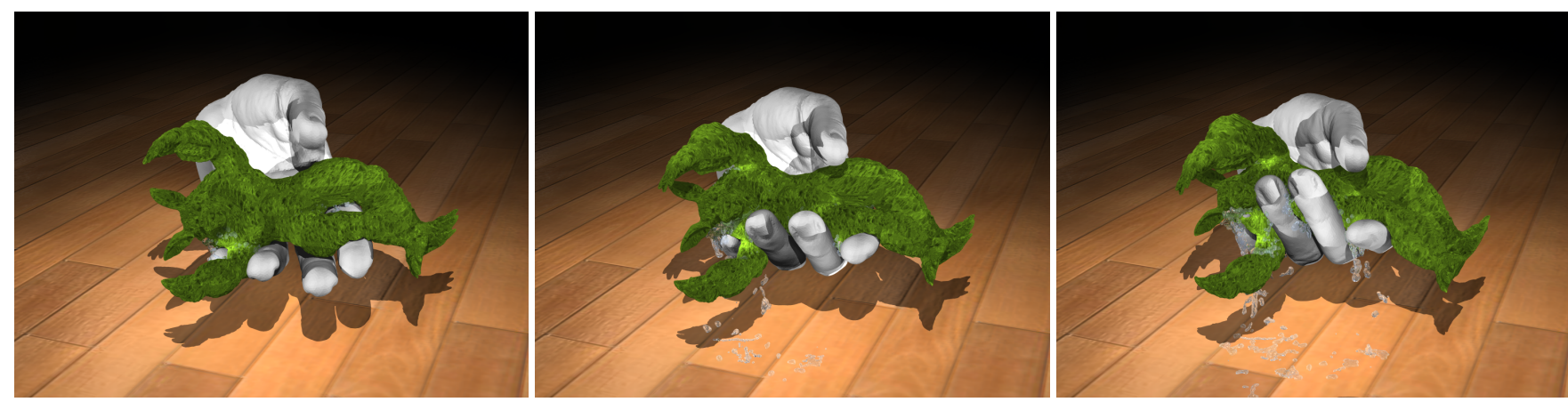

Figure 7: A wet porous Armadillo model is squeezed in a hand. The pressure on the pores pushes the fluid outwards.
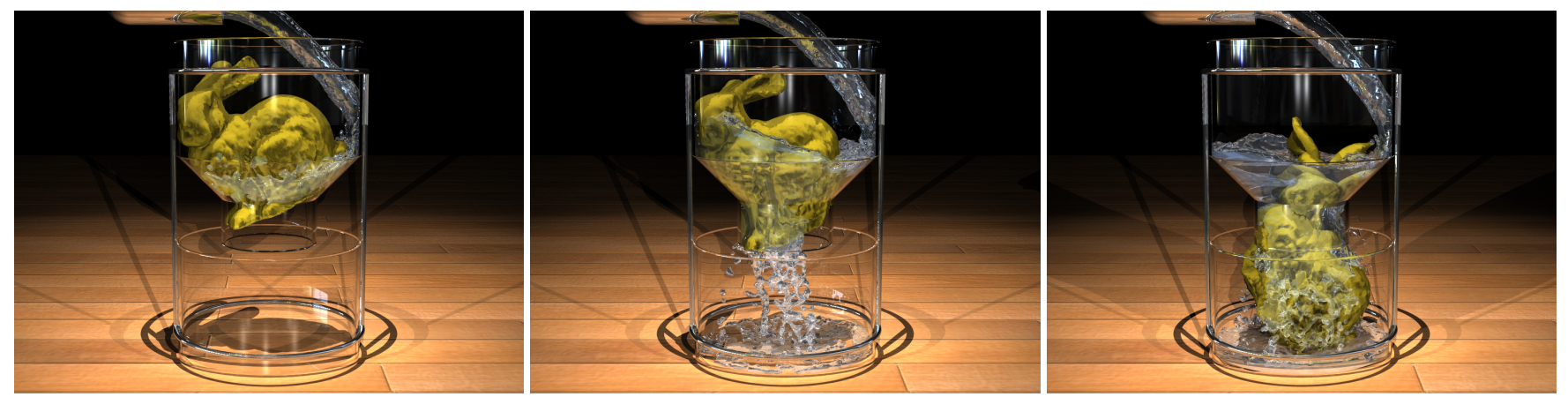

Figure 8: A dry porous Stanford Bunny is dropped in a funnel. As water is absorbed the porous material weakens and the Bunny sags through the funnel.

BECKER, M., AND TESCHNER, M. 2007. Weakly compressible SPH for free surface flows. In SCA '07: Proceedings of the 2007 ACM SIGGRAPH/Eurographics symposium on Computer animation, 209-217.

Bell, N., Yu, Y., AND Mucha, P. J. 2005. Particle-based simulation of granular materials. In SCA '05: Proceedings of the 2005 ACM SIGGRAPH/Eurographics symposium on Computer animation, ACM Press, New York, NY, USA, 77-86.

Berry, R. A., Martineau, R. C., And Wood, T. R. 2004 Particle-based direct numerical simulation of contaminant transport and deposition in porous flow. Vadose Zone Journal 3, 1 (February), 164-169.

Carlson, M., Mucha, P. J., And Turk, G. 2004. Rigid fluid: animating the interplay between rigid bodies and fluid. $A C M$ Trans. Graph. 23, 3, 377-384.

ChU, N. S.-H., AND TAI, C.-L. 2005. Moxi: real-time ink dispersion in absorbent paper. In SIGGRAPH '05: ACM SIGGRAPH 2005 Papers, ACM Press, New York, NY, USA, 504-511.

Clavet, S., Beaudoin, P., And Poulin, P. 2005. Particlebased viscoelastic fluid simulation. In SCA '05: Proceedings of the 2005 ACM SIGGRAPH/Eurographics symposium on Computer animation, ACM Press, New York, NY, USA, 219-228.

Cuesta, C., van Duijn, C., And Hulshof, J. 1999. Infiltration in porous media with dynamic capillary pressure: travelling waves. Tech. Rep. MAS-R9932, CWI, Nov.

DARCy, H. 1856. Les Fontaines Publiques de la Ville de Dijon, Dalmont, Paris.
Desbrun, M., And CANI, M.-P. 1999. Space-time adaptive simulation of highly deformable substances. Tech. Rep. 3829, INRIA, BP 105 - 78153 Le Chesnay Cedex - France, December.

Desbrun, M., And Gascuel, M.-P. 1996. Smoothed particles: A new paradigm for animating highly deformable bodies. In Computer Animation and Simulation '96, 61-76.

Foster, N., AND FEDKIW, R. 2001. Practical animation of liquids. In SIGGRAPH '01: Proceedings of the 28th annual conference on Computer graphics and interactive techniques, ACM, New York, NY, USA, 23-30.

Foster, N., And Metaxas, D. 1996. Realistic animation of liquids. Graph. Models Image Process. 58, 5, 471-483.

Foster, N., ANd Metaxas, D. 1997. Controlling fluid animation. In CGI '97: Proceedings of the 1997 Conference on Computer Graphics International, IEEE Computer Society, Washington, DC, USA, 178.

Gingold, R. A., And Monaghan, J. J. 1977. Smoothed particle hydrodynamics: theory and application to non-spherical stars. Mon. Not. R. astr. Soc. 181, 375-389.

Guendelman, E., Selle, A., Losasso, F., And Fedkiw, R. 2005. Coupling water and smoke to thin deformable and rigid shells. In SIGGRAPH '05: ACM SIGGRAPH 2005 Papers, ACM Press, New York, NY, USA, 973-981.

Harada, T., Koshizuka, S., And Kawaguchi, Y. 2007. Smoothed particle hydrodynamics on GPUs. In Computer Graphics International, 63-70.

Hegeman, K., Carr, N. A., And Miller, G. S. 2006. Particlebased fluid simulation on the GPU. In Computational Science- 
ICCS 2006, Springer, P. M. S. Vassil N. Alexandrov, Geert Dick van Albada and J. Dongarra, Eds., vol. 3994 of LNCS, 228-235.

HilfER, R. 1996. Transport and relaxation phenomena in porous media. Advances in Chemical Physics XCII, 299.

Hilfer, R. 2006. Macroscopic capillarity and hysteresis for flow in porous media. Physical Review E (Statistical, Nonlinear, and Soft Matter Physics) 73, 1, 016307.

Jensen, H. W., Legakis, J., And Dorsey, J. 1999. Rendering of wet materials. In Rendering Techniques, Proceedings of the Eurographics Workshop on Rendering, 273-282.

Keiser, R., Adams, B., Gasser, D., Bazzi, P., Dutré, P., AND GROss, M. 2005. A unified Lagrangian approach to solidfluid animation. In Proceedings of the Eurographics Symposium on Point-Based Graphics, 125-133.

Keiser, R., Adams, B., Dutré, P., Guibas, L. J., And Pauly, M. 2006. Multiresolution particle-based fluids. Tech. rep., ETH Zurich.

Kipfer, P., And Westermann, R. 2006. Realistic and interactive simulation of rivers. In Proceedings Graphics Interface 2006, Canadian Human-Computer Communications Society, S. Mann and C. Gutwin, Eds., 41-48.

Kolb, A., And Cuntz, N. 2005. Dynamic particle coupling for GPU-based fluid simulation. In Proc. 18th Symposium on Simulation Technique, 722-727.

LEKNER, J., AND DORF, M. C. 1988. Why some things are darker when wet. Applied Optics 27, 7, 1278-1280.

Lorensen, W. E., And Cline, H. E. 1987. Marching cubes: A high resolution $3 \mathrm{~d}$ surface construction algorithm. In $S I G$ GRAPH 87: Proceedings of the 14th annual conference on Computer graphics and interactive techniques, ACM Press, New York, NY, USA, 163-169.

LUCY, L. B. 1977. A numerical approach to the testing of the fission hypothesis. Astronomical Journal 82 (Dec.), 1013-1024.

Monaghan, J. 1992. Smoothed particle hydrodynamics. Annual Revision on Astronomy and Astrophysics 30, 543-574.

Monaghan, J. J. 2005. Smoothed particle hydrodynamics. Reports on Progress in Physics 68, 8 (August), 1703-1759.

Morris, J. P., Fox, P. J., AND ZHU, Y. 1997. Modeling low reynolds number incompressible flows using SPH. J. Comput. Phys. 136, 1, 214-226.

Morris, J. P., ZHU, Y., AND Fox, P. J. 1999. Parallel simulations of pore-scale flow through porous media. Computers and Geotechnics 25, 4, 227-246.

Müller, M., Charypar, D., And Gross, M. 2003. Particlebased fluid simulation for interactive applications. In $S C A$ '03: Proceedings of the 2003 ACM SIGGRAPH/Eurographics symposium on Computer animation, Eurographics Association, Airela-Ville, Switzerland, Switzerland, 154-159.

Müller, M., Keiser, R., Nealen, A., Pauly, M., Gross, M., AND AleXA, M. 2004. Point based animation of elastic, plastic and melting objects. Proceedings of 2004 ACM SIGGRAPH Symposium on Computer Animation, 141-151.

Müller, M., Schirm, S., Teschner, M., Heidelberger, B., AND GROSS, M. H. 2004. Interaction of fluids with deformable solids. Journal of Visualization and Computer Animation 15, 3-4, 159-171.

Müller, M., Solenthaler, B., Keiser, R., And Gross, M. 2005. Particle-based fluid-fluid interaction. In SCA '05:

ACM Transactions on Graphics, Vol. 27, No. 3, Article 49, Publication date: August 2008
Proceedings of the 2005 ACM SIGGRAPH/Eurographics symposium on Computer animation, ACM Press, New York, NY, USA, 237-244.

NitAo, J. J., AND BEAR, J. 1996. Potentials and their role in transport in porous media. Water Resources Research 32, 225250 .

Premoze, S., Tasdizen, T., Bigler, J., Lefohn, A., And Whitaker, R. T. 2003. Particle-based simulation of fluids. Eurographics 2003 / Computer Graphics Forum 22, 3, 401-410.

SaWley, M., Cleary, P., AND Ha, J. 1999. Modelling of flow in porous media and resin transfer moulding using smoothed particle hydrodynamics. In Second International Conference on CFD in the Minerals and Process Industries, 473-478.

Scheidegger, A. E. 1957. The Physics of Flow through Porous Media. University of Toronto Press and Oxford University Press.

Solenthaler, B., Schläfli, J., and Pajarola, R. 2007. A unified particle model for fluid-solid interactions. Computer Animation and Virtual Worlds 18, 1, 69-82.

STAM, J. 1999. Stable fluids. In SIGGRAPH '99: Proceedings of the 26th annual conference on Computer graphics and interactive techniques, ACM Press/Addison-Wesley Publishing Co., New York, NY, USA, 121-128.

Twomey, S. A., Bohren, C. F., And Mergenthaler, J. L. 1986. Reflectance and albedo differences between wet and dry surfaces. Applied Optics 25 (feb), 431-437.

ZHU, Y., AND FoX, P. J. 2001. Smoothed particle hydrodynamics model for diffusion through porous media. Transport in Porous Media 43, 3 (June), 441-471.

ZHU, Y., AND FOX, P. J. 2002. Simulation of pore-scale dispersion in periodic porous media using smoothed particle hydrodynamics. J. Comput. Phys. 182, 2, 622-645.

ZHU, Y., FoX, P. J., AND MORRIS, J. 1999. A pore-scale numerical model for flow through porous media. International journal for numerical and analytical methods in geomechanics 23, 9, 881-904. 